\title{
Implications of Point Defects on the Atomic Structure of Domain Walls in $\mathrm{BiFeO}_{3}$
}

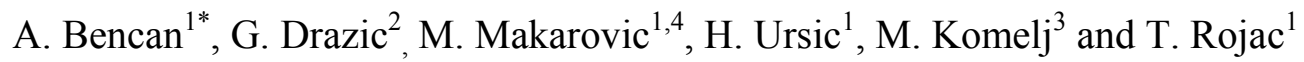 \\ ${ }^{1 .}$ Electronic Ceramics Department, Jozef Stefan Institute, Ljubljana, Slovenia. \\ 2. Department of Materials Chemistry, National Institute of Chemistry, Ljubljana, Slovenia. \\ 3. Department for Nanostructured Materials, Jozef Stefan Institute, Ljubljana, Slovenia. \\ 4. Jozef Stefan International Postgraduate School, 1000 Ljubljana, Slovenia. \\ * Corresponding author: andreja.bencan@ijs.si
}

Ferroelectric and ferroelastic domain walls (DWs) are planar topological defects, which separate two domain regions with uniform polarization. DWs may form along various crystallographic planes, may accumulate different ionic or electronic point defects, and may be pinned by point defects, dislocations or grain boundaries. All these structural features, which are set by the way of how the material is synthesized, have an influence on the DW properties, such as DW conductivity in ferroelectric $\mathrm{BiFeO}_{3}$ (BFO), and are thus directly exploitable in the so-called "domain-wall nanoelectronics" $[1,2,3]$ or may indirectly influence the functional response of polycrystalline ferroelectrics [4]. To make further steps in tailoring the DW properties, in-depth understanding of the structural details of DWs on the nano and atomic scales are needed.

This study is the next step that follows on from our recent publication on domain-wall conductivity in BFO [5] were we explained the extrinsic nature of the DW conduction mechanism dominated by the segregation of charged defects (Bi vacancies and $\mathrm{Fe}^{4+}$ ) at the DWs. Using Scanning Transmission Electron Microscopy (STEM) coupled with Electron Energy Loss Spectroscopy (EELS), we show in this presentation that through different material processing conditions (temperature, atmosphere, and cooling rates) we may control the type, and concentration of defects (bismuth and oxygen vacancies, electron holes) at DWs and consequently tailor the local DW structure, thickness, unit-cell distortions across DW and thus DW conductivity.

Using the same methodology as in [5], we experimentally determine the type of defects and their effect on the DW thickness and lattice strain distribution inside and outside the DW region. By measuring the cation/anion displacements from STEM images, with a resolution down to $\sim 5$ pm we identify the position and the type (charged/non-charged) of DWs. We quantitatively determine strains by peak-pair analysis and identify point defects (such as $\mathrm{Bi}$ vacancies) from relative intensity distribution of the atomic columns in high-angle annular dark field images. EELS analysis is used for the determination of the chemical composition and the valence state of Fe ion at the atomic level. The DW's local conductivity is analyzed using conductive atomic force microscopy.

Figure 1 illustrates a neutral DW (a) and a charged DW (d) exhibiting particular strain inside the DW (f). Neutral DW is bismuth vacancies - free (b). The lower Bi column intensities (e) suggest accumulation of bismuth vacancies in the charged DW having large influence on the distortion of the unit-cells at DW (f). In the presentation, defects contributing to the structural variations at DWs supported by ab-initio structure calculations will be discussed. 
References:

[1] J. Seidel et al., Nat. Mater. 8 (2009), p.229.

[2] P. Sharma et al., Sci. Adv. 3 (2017), e1700512.

[3] G. Catalan et al., Rev. Mod. Phys. 84 (2012), p.119.

[4] T. Rojac et al., Adv. Funct. Mater. 25 (2015), p.2099.

[5] T. Rojac et al., Nat. Mat. 16 (2017), p.322.

[6] This work was supported by the Slovenian Research Agency within programs (P2-0105, P2-0393) and projects J2-9253, PR-08298.
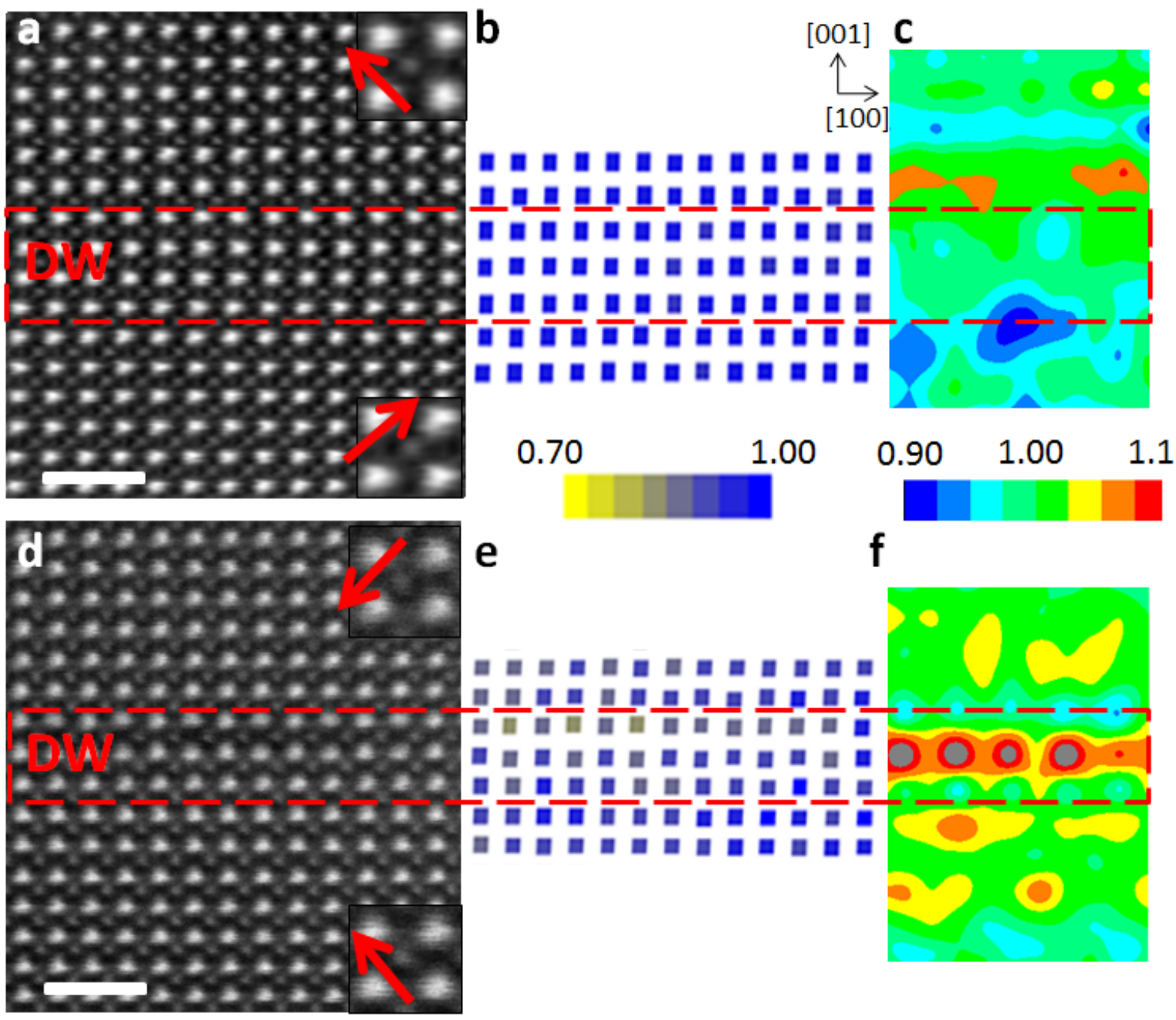

Figure 1. HAADF images of neutral (head-to-tail) (a) and charged (tail-to-tail) (d) $109^{\circ}$ DWs in [010] zone axis with the corresponding normalized distribution map of Bi column intensities across DW (b,e) and c/a lattice map (c,f). The insets of panels $(\mathrm{a}, \mathrm{d})$ show the Fe displacement directions in the two adjacent domains, the red boxes indicate the DW regions. Scale bar represents $1 \mathrm{~nm}$. 